\title{
Stellenwert bei der Allokation der Nierenexplantate
}

Quelle: Kasiske BL, Stewart DE, Bista BR et al. The role of procurement biopsies in acceptance decisions for kidneys retrieved for transplant. Clin J Am Soc Nephrol 2014; 9: 562-571

Thema: In Anbetracht des Mangels an postmortalen Spendernieren würden viele Patienten auf der Warteliste offensichtlich von den Organen profitieren, die als „non transplantable“ abgelehnt werden. So sind in den USA die Ergebnisse einer routinemäßig durchgeführten Nierenbiopsie die häufigste Ursache für eine Ablehnung der postmortal explantierten Donornieren. Die vorliegende Studie verglich u.a. die Biopsieergebnisse von abgelehnten Nierenexplantaten mit den Biopsieergebnissen von den (kontralateralen) Nieren, die erfolgreich transplantiert wurden.

Projekt: Die vorliegende prospektive, fallkontrollierte Beobachtungsstudie analysierte die Biopsieergebnisse von 83 Nie- ren, die im Jahre 2010 aufgrund von Biopsieergebnissen abgelehnt wurden (vs. kontralaterale Explantate, welche transplantiert wurden), sowie Nierenbiopsien von 83 verstorbenen Patienten (keine Nierenspender), welche im Hinblick auf das Spender-Risiko-Profil des Vergleichskollektivs identisch waren („randomlymatched"-Kontrolle).

Ergebnisse: Die Aussagekraft der Biopsiebefunde bzgl. der Transplantabilität war unzureichend. Der Umfang von tubulären Atrophien, interstitiellen Entzündungen, arteriolären Hyalinosen und akuten Tubulusnekrosen wurde häufig nicht angegeben (94\% Stanzbiopsien). Der prozentuale Anteil der Glomerulosklerose zeigte eine erhebliche Überlappung bei den untersuchten Fällen sowie zwischen kontralateralen Kontrollen und Kontrollen: $17,1 \% \pm 15,3 \%, 9,0 \pm 6,6 \%$ und $5,0 \% \pm 5,9 \%$. Von allen Biopsieergebnissen korrelierte nur die Glomerulosklerose mit der

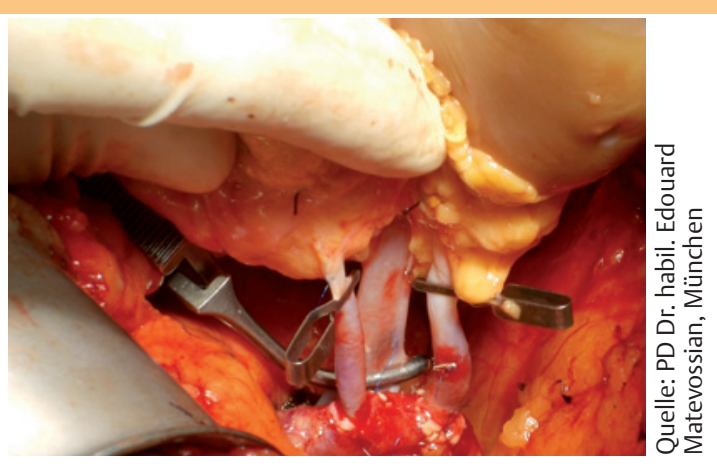

Organablehnungsrate (Odds Ratio 15,09; 95-\%-Konfidenzintervall 2,47 zu 92,41; $\mathrm{P}=0,003)$ als indirekter Hinweis darauf, dass nur dieses Ergebnis während der Allokation bei dem Entschluss zu einer Annahme/Ablehnung des Organs berücksichtigt wurde.

Fazit: Die nicht ausreichend spezifisch histopathologisch untersuchten Routinebiopsien der postmortal entnommenen Nieren können zu Organablehnung seitens des Transplantationszentrums führen, welche aus der retrospektiven Sicht in den meisten Fällen nicht erforderlich war.

Schlüsselwörter: Biopsie - postmortale Organspende - Organqualität Nierenexplantation

PD Dr. habil. Edouard Matevossian, München

Kommentar

Die vorliegende retrospektive Fall-Kontroll-Studie in der Zeit der persistierenden Organknappheit sensibilisiert uns (die klinisch tätigen Transplantationsmediziner) für die Frage nach der „Eignung des Explantates zur Transplantation“ und betrifft nur die postmortale Organspende. Hierbei wird in der Diskussion interdisziplinär konkludiert, dass der Einsatz von Routinebiopsien den Organverlust prinzipiell reduzieren würde, was aus den Daten der Studie sowie aus der klinischempirischen Sicht eine plausible Konklusion ist. Andererseits: Eine Routinebiopsie zur Evaluation des Organs bzgl. der Transplantabilität würde bei unzureichender spezifischer „isolierter“ Wertung des Befundes zur Steigerung der Transplantationsablehnungsrate führen.

Kritisch, vor allem aus der Sicht des Klinikers, welcher als verantwortlicher Chirurg sowohl an Explantationen als auch an Organtransplantationen beteiligt ist, anzumerken sind die fehlenden Angaben seitens des Explantationsteams zur morphologisch-anatomischen Organ- qualität (Organreport) sowie die relativ „unscharfen“ Angaben der histopathologischen Biopsieauswertung [1]. Hier wäre es hoch interessant, die Korrelation zwischen den im Organreport dokumentierten Angaben (welche an die zentrale Institution, ähnlich wie Eurotransplant, geleitet werden, sodass mit dieser Information die Transplantationszentren des potenziellen Empfängers konfrontiert werden) und der retrospektiven histopathologischen Wertung seitens eines Pathologie-Referenz-Zentrums zu erforschen, wenngleich sich dies lediglich semiquantitativ machen lässt [1, 2].

Abgesehen von den obigen editorialen Kommentaren und theoretisch extrapoliert auf den Eurotransplantraum könnten bestimmte ergänzende Maßnahmen bei der Organdokumentation bei klinisch verifizierten fraglichen Befunden einen sinnvollen Beitrag zur Evaluation der Transplantabilität leisten. Dazu gehört die relevante Fotodokumentation mit adäquatem Kommentar, welche ohne nennenswerten Aufwand mitsamt des
Organreports an Eurotransplant zur Allokation vermittelt werden kann. Dies würde zweifelsohne die initiale Ablehnungsrate der Nierenexplantate, bedingt v.a. durch die alleinige deskriptive Dokumentation im Organreport, reduzieren und den Allokationsprozess optimieren.

Eine definitive, wissenschaftlich fundierte Evaluation der Überlegenheit dieser klinisch orientierten logistischen Vorgehensweise bleibt durch eine prospektive Multicenterstudie unter Beteiligung der regionalen DSO-Zentren sowie von Eurotransplant zu klären. Die Kosten-NutzenRelation ist vertretbar und wird sicher bei der obigen klinisch und patientenorientierten Zielsetzung lediglich eine untergeordnete Rolle spielen.

PD Dr. habil. Edouard Matevossian, München

Literatur

1 Loss $M$, Bald C, Breidenbach $T$ et al. [Abdominal organ retrieval: strategies to improve quality]. Chirurg 2013; 84: 263270

2 Guba M, Bald C, Kleespies A et al. [Surgical quality of organ retrieval in Bavaria]. Chirurg 2013; 84: 271-276 